\title{
\begin{tabular}{|l|l|l|l|l}
\hline $\mathrm{M}$ & $\mathrm{R}$ & $\mathrm{S}$ & Internet Journal of & Nitride Semiconductor Research \\
\hline
\end{tabular}
}

Volume 2, Article 10

\section{Photoluminescence excitation spectroscopy of GaN thin layers as a function of temperature}

\author{
C. Guénaud, E. Deleporte, M. Voos, C. Delalande \\ Laboratoire de Physique de la Matière Condensée, École Normale Superièure, Paris, France \\ B. Beaumont, M. Leroux, Pierre Gibart, J. P. Faurie \\ Centre de Recherche sur l'Hetero-Epitaxie et ses Applications, CRHEA-CNRS
}

This article was received on June 10, 1997 and accepted on July 9, 1997.

\begin{abstract}
We report on photoluminescence and photoluminescence excitation experiments performed on hexagonal GaN layers grown on a Sapphire substrate. Information about extrinsic and intrinsic optical properties have been obtained. We show that, at low temperature, the fundamental A excitons are preferentially involved in the relaxation towards the neutral donor bound exciton photoluminescence line, while electron-hole pairs rather participate in the relaxation towards $D^{0}-A^{0}$ emission and the yellow band. The relaxation from the $A$ exciton towards the yellow band and $D^{0}-A^{0}$ emission is made easier by temperature. The band structure of the GaN layers has been determined from temperature dependent photoluminescence excitation spectroscopy: $A$ and $C$ excitons and $A$ continuum band gap have been identified up to $210 \mathrm{~K}$.
\end{abstract}

\section{Introduction}

GaN-based III-V nitride semiconductors have recently attracted great attention because they are involved in many applications: high-temperature electronics, light emitters and detectors operating in the blue and ultra-violet wavelength range. The potentiality of nitrides for short wavelength light emitting devices is now proved, since the recent attainment of the first laser diode based on wide band gap III-V nitrides by $\mathrm{S}$. Nakamura [1]. The application of $\mathrm{GaN}$ for optoelectronic devices requires a detailed knowledge of its fundamental optical properties (for a review, see [2]). To optimize the efficiency of the laser devices, both extrinsic and intrinsic optical properties have to be explored. Photoluminescence [3] [4] [5] [6] [7] [8] [9] yields information mainly about extrinsic optical properties (yellow band, $D^{0}-A^{0}$ emission) whereas photoluminescence excitation spectroscopy [10] [11], reflectivity [4] [6] [7] [9] [12] [13] and absorption [4] [14] allow one to obtain information about the intrinsic excitonic structure of GaN. Unfortunately, due to the lack of large GaN substrates of good crystalline quality, $\mathrm{GaN}$ layers are usually grown on foreign substrates $\left(\mathrm{Al}_{2} \mathrm{O}_{3}, 6 \mathrm{H}-\mathrm{SiC}\right.$, $\mathrm{GaAs}$ ), inducing strong strains in the layer. It turns out that intrinsic optical properties are very sensitive to strains [12] [13] [15]. Thus the assignment of the observed peaks is not easy, especially since the intrinsic parameters of GaN, such as effective masses, exciton binding energies, dielectric constants, strain parameters (elastic constants, deformation potentials) are not well known [16], despite recent pressure-dependent [17] and temperature-dependent [8] [9] [14] [18] [19] [20] optical experiments and calculations [21] [22].

From photoluminescence (PL) results combined with photoluminescence excitation (PLE) experiments performed on hexagonal GaN thin layers grown on a Sapphire substrate, we get information about the relaxation dynamics towards the impurity related $\mathrm{PL}$ lines. At low temperature, the fundamental $A$ excitons are involved in the relaxation towards the neutral donor bound exciton PL line, but they are only weakly involved in the relaxation towards $\mathrm{D}^{0}-\mathrm{A}^{0}$ donor-acceptor pair emission and yellow band. The electron-hole pairs are preferentially involved in the relaxation towards $D^{0}-A^{0}$ emission and the yellow band. We show that the relaxation from the $A$ exciton towards the yellow band and $D^{0}-A^{0}$ emission, which is difficult at low temperature, is made easier by temperature. From PLE spectroscopy performed as a function of temperature, information 
about the intrinsic excitonic properties of GaN layers are obtained: the $A$ and $C$ excitons have been identified up to $210 \mathrm{~K}$. Our results are consistent with an A exciton binding energy of $29 \mathrm{meV}$.

\section{Experimental results.}

\subsection{Experimental set-up}

Two undoped hexagonal GaN films were prepared by organo-metallic vapor phase epitaxy [6] [7] [23] on (0001) sapphire substrates with the c-axis parallel to the growth axis. The samples I and II are 17 micrometers and 7 micrometers thick respectively. In our PL and PLE experiments, excitation is provided by a cw Xe $450 \mathrm{~W}$ short arc lamp, whose wavelength is selected by a $\mathrm{f}=32 \mathrm{~cm}$ double monochromator. The photoluminescence signal is analyzed through a $\mathrm{f}=1 \mathrm{~m}$ double monochromator by a Hamamatsu R212 photomultiplier, followed by standard lock-in technics. A reference signal is simultaneously stored which allows a correction of the PLE signal. The energy resolution of our system is $2 \mathrm{meV}$, for an excitation intensity of several $\mathrm{mW}^{-\mathrm{cm}^{-2}}$. The temperature can be varied from $4 \mathrm{~K}$ to $300 \mathrm{~K}$.

\subsection{Low temperature luminescence and luminescence excitation spectra.}

The PL spectrum of sample I at low temperature $(T=2 \mathrm{~K})$ is shown in figure 1 , the excitation energy is $4 \mathrm{eV}$. In the following, we will present experimental results on sample I only, as results on sample II are very similar. Several emission bands are observed. The very broad band peaking around $2.3 \mathrm{eV}$ is the yellow band [4] [5]. We detect at $3267 \mathrm{meV}$ the donor-acceptor pairs $\left(\mathrm{D}^{0}-\mathrm{A}^{0}\right)$ recombination involving the usual residual donors and acceptors of GaN [2]. Two phonon replica can be seen at $3175 \mathrm{meV}$ and $3083 \mathrm{meV}$ (the energy of the $\mathrm{LO}$ phonon is $\mathrm{h} \omega_{\mathrm{LO}}=92 \mathrm{meV}$ [24]). The whole spectrum is dominated by band edge transitions involving the band and free fundamental A exciton [3] [4] [6] [7]. Figure 1b shows the details of the PL spectrum in the energy range 3.4-3.5 eV. Two peaks are observed at $3457 \mathrm{meV}$ and $3474 \mathrm{meV}$, and a shoulder is seen at $3482 \mathrm{meV}$. An increase of the temperature leads to an efficient quenching of the two lower energy peaks and a great increase of the shoulder, which becomes the only observed peak at $T>100 \mathrm{~K}$. Due to temperature induced detrapping of excitons from impurities, only free excitons are observable at high temperature. So we interpret the peak at $3457 \mathrm{meV}$ as an acceptor bound exciton $\mathrm{I}_{1}$, the peak at $3474 \mathrm{meV}$ as a donor bound exciton $\mathrm{I}_{2}$, and the shoulder at $3482 \mathrm{meV}$ as the fundamental intrinsic A exciton (this will be confirmed by PLE spectra). A phonon replica of $\mathrm{I}_{2}$ can be seen at $3380 \mathrm{meV}$ [2] [4] [6] [7].

At low temperature, the ratio of the $\mathrm{I}_{2}$ peak intensity to the yellow band peak intensity or the $\mathrm{D}^{0}-\mathrm{A}^{0}$ emission intensity is about $10^{3}$. Band edge excitonic emission is thus much stronger than impurity related emissions, indicating the good quality of our samples.

Figure 2 shows the PLE spectra at $30 \mathrm{~K}$, with different detection energies: $\mathrm{I}_{2}, \mathrm{D}^{0}-\mathrm{A}^{0}$, and the yellow band. The $A$ exciton at $3479 \mathrm{meV}$ and an excited excitonic transition at $3498 \mathrm{meV}$ (see below for its interpretation) are very well observed in the PLE spectrum detected at $\mathrm{I}_{2}$. On the contrary, A exciton appears very weakly in the PLE

spectra when we detect at $D^{0}-A^{0}$ and at the yellow band. This suggests that the $A$ exciton absorption preferentially promotes the $\mathrm{I}_{2}$ luminescence. At high energies (far above $3.5 \mathrm{eV}$ ), in the electron-hole pairs states, we still get signal when we detect energy at the $D^{0}-A^{0}$ line or the yellow band. However, we measure very weak luminescence when the detection energy is set to the $I_{2}$ line (see figure 2). We may deduce that e-h pairs are preferentially involved in the relaxation towards the $D^{0}-A^{0}$ line and the yellow band.

\subsection{Luminescence and luminescence excitation spectra versus temperature.}

The PL spectra for 30 and $150 \mathrm{~K}$ are reported in figure 3 . As temperature increases, the $\mathrm{A}$ exciton PL line intensity decreases for the benefit of the $D^{0}-A^{0}$ line and the yellow band. The yellow band luminescence intensity turns out to be the strongest at high temperatures. Figure 4 exhibits the PLE spectra of the yellow band versus temperature: the $A$ exciton line becomes increasingly intense as temperature increases. We conclude that the relaxation from the $A$ exciton towards the $D^{0}-A^{0}$ transition and the yellow band, which appears to be very difficult at low temperature (see figure 2), is made easier by temperature.

We first interpret the excited line we detect at $3498 \mathrm{meV}(30 \mathrm{~K})$. Following former PLE measurements [5] [10], we may assume this is the $\mathrm{C}$ exciton line. However, many experimental results based on reflectivity (REF) and 
strain determination [12] [13] [15], have been obtained. These REF-based results suggest this line could also be the excited A(2s) (as it is, most of the authors reinterpret Monemar's $C$ line [10] as being the A(2s)).

Let us first assume this excited transition ( $3498 \mathrm{meV}$ at $30 \mathrm{~K}$ ) is the $\mathrm{A}(2 \mathrm{~s})$ line. As the $\mathrm{A}$ line lies at $3479 \mathrm{meV}$ at the same temperature, we would end up with an A binding energy of $25.3 \mathrm{meV}$. This would be in agreement with other experimental results which yield the A exciton binding energy between $20 \mathrm{meV}$ [25] [26] [27] and $28 \mathrm{meV}[18]$.

Figure 5 exhibits the energy positions of the fundamental exciton A and of the first excited excitonic transition for temperatures from $30 \mathrm{~K}$ to $250 \mathrm{~K}$ as measured by our PLE experiments (detection is set to the yellow band). We see the A excitonic transition energy decreasing from $3479 \mathrm{meV}$ to $3436 \mathrm{meV}$, while the first excited excitonic transition energy decreases from $3498 \mathrm{meV}$ to $3472 \mathrm{meV}$, as $\mathrm{T}$ is tuned from $30 \mathrm{~K}$ to $210 \mathrm{~K}$. This implies that the energy difference $A(2 s, T)-A(T)$ ranges from 19 to $36 \mathrm{meV}$ within this temperature variation. That is, the Rydberg energy would change with temperature. This could only be explained by a thermally induced modification of the reduced masses, this modification being actually caused by thermally induced strains. Shikanai et al [13] have shown that the reduced effective masses, and thus the binding energy of the $A$ exciton does not depend on strains: therefore, $A(2 s, T)-A(T)$ cannot vary with temperature. We conclude that the excited feature we observe must be attributed to the $C$ exciton line.

We still have to explain why the energy difference $A$ - C changes with temperature. The temperature dependence of the excitonic energies in a bulk [14] [28] comes from electron-phonon interactions [28] and thermal expansion effect, which accounts for the effect of the change of lattice constants on the energy gap. Measurements and calculations of $A$ and $C$ energy positions versus temperature in a GaN bulk are not yet available to our knowledge. However, looking at results on other würtzite materials like Zn0, CdS or CdSe bulks [29], we see that the evolutions of $A$ - C energy versus temperature are practically parallel and we may expect the same tendency for GaN bulks. We thus need another feature to explain the A - C variation with temperature. In our case of a GaN layer grown onto a Sapphire substrate, we must also take into account the different thermal expansion coefficients of these two materials, which will induce additional strains in the GaN layer. Taking a rough evaluation of the thermal expansion coefficients of $\mathrm{GaN}: \alpha_{\mathrm{GaN}}=5.6 .10^{-6} \mathrm{~K}^{-1}$, and of Sapphire: $\alpha_{\text {Sapph }}=7.5 .10^{-6} \mathrm{~K}^{-1}$ [9], we find that varying the temperature from $2 \mathrm{~K}$ to $250 \mathrm{~K}$ induces a strain of the order of magnitude of $0.05 \%$ in the GaN layer. Shikanai et al [13] have studied by reflectivity several samples with various strains. If we look at their A-C energy positions versus strain, we see that a variation of $0.05 \%$ will cause $A-C$ to change by about $15 \mathrm{meV}$. This is in reasonable agreement with our results since in our case the shift from 30 to $210 \mathrm{~K}$ (inducing a strain evaluated to $0.05 \%$ ) causes $A-C$ to vary by $17 \mathrm{meV}$. We would like to emphasize that this is only an evaluation: to explain quantitatively the different temperature evolutions of $A$ and $C$ excitons, we need to know the strain tensor, the deformations potentials, the thermal dilatation coefficients (and mostly their dependence on temperature), but these data are not available in literature yet.

Following Monemar's idea, we may interpret the small dip in figure $4(30 \mathrm{~K})$ at $3508 \mathrm{meV}$ as the beginning of the band to band absorption. This would yield a binding energy of $29 \mathrm{meV}$ for the A exciton. This value may be debated as PLE experiments are not a straightforward way to determine the Rydberg energy: a fit of the absorption coefficient (including the electron-hole correlation effects) and of the relaxation mechanism would be necessary.

\section{Conclusion}

The relaxation dynamics towards the impurity related PL lines have been studied. At low temperature, the fundamental $A$ excitons are preferentially involved in the relaxation towards the neutral donor bound exciton $P L$ line, while electron-hole pairs rather participate in the relaxation towards $D^{0}-A^{0}$ emission and the yellow band. We have shown that the relaxation from $A$ exciton towards the yellow band and $D^{0}-A^{0}$ emission, which is difficult at low temperature, is made easier by temperature.

PLE spectroscopy performed as a function of temperature up to $250 \mathrm{~K}$ has allowed the assignment of the observed excitonic peaks. The first excited excitonic transition, whose energy is $19 \mathrm{meV}$ higher than $\mathrm{A}$ exciton transition energy at $30 \mathrm{~K}$ corresponds to the $\mathrm{C}$ excitonic transition. The exciton binding energy is estimated to be of the order of $29 \mathrm{meV}$. A and $\mathrm{C}$ excitons have different selection rules and polarization experiments have to be performed to complete our results.

\section{Acknowledgments}

This work is supported by an European Community contract: ESPRIT-LTR LAQUANI no20968. 


\section{References}

[1] Shuji Nakamura, Masayuki Senoh, Shin-ichi Nagahama, Naruhito Iwasa, Takao Yamada, ToshioMatsushita, Hiroyuki Kiyoku, Yasunobu Sugimoto , Appl. Phys. Lett. 68, 2105-2107 (1996).

[2] S. Strite, H. Morkoç, J. Vac. Sci. Technol. B 10, 1237-1266 (1992).

[3] J. I. Pankove, J. E. Berkeyheiser, H. P. Maruska, J. Wittke, Sol. St. Comm. 8, 1051 (1970).

[4] R. Dingle, D. D. Sell, S. E. Stokowski, M. Ilegems, Phys. Rev. B 4, 1211 (1971).

[5] T. Ogino, M. Aoki, Jpn. J. Appl. Phys. 19, 2395 (1980).

[6] M. Leroux, B. Beaumont, N. Grandjean, Pierre Gibart, J. Massies, J. P. Faurie, MRS Internet J. Nitride Semicond. Res. 1, 25 (1996).

[7]M. Leroux, B. Beaumont, N. Grandjean, C. Golivet, P. Gibart, J. Massies, J. Leymarie, A. Vasson, A.AM. Vasson, "Comparative optical characterization of GaN grown by metalorganics vapor phase epitaxy (MOVPE), Gas source molecular beam epitaxy (GSMBE) and Halide vapor phase epitaxy (HVPE)", Proceedings of the EMRS Spring Meeting, Strasbourg (1996)

[8] B. Monemar, J. P. Bergman, I. A. Buyanova, W. Li, H. Amano, I. Akasaki, MRS Internet J. Nitride Semicond. Res. 1, 2 (1996).

[9] IA Buyanova, JP Bergman, B Monemar, H Amano, I Akasaki, Appl. Phys. Lett. 69, 1255-1257 (1996).

[10] B. Monemar, Phys. Rev. B 10, 676 (1974).

[11] D Kovalev, B Averboukh, D Volm, BK Meyer, H Amano, I Akasaki, Phys. Rev. B 54, 2518-2522 (1996).

[12] B Gil, O Briot, RL Aulombard, Phys. Rev. B 52, R17028-17031 (1995).

[13] A Shikanai, T Azuhata, T Sota, S Chichibu, A Kuramata, K Horino, S Nakamura, J. Appl. Phys. 81, 417-424 (1997).

[14] H. Teisseyre, P. Perlin, T. Suski, I. Grzegory, S. Porowski, J. Jun , A. Pietraszko , T. D. Moustakas , J. Appl. Phys. 76, 2429-2434 (1994).

[15] D Volm, K Oettinger, T Streibl, D Kovalev, M Ben-Chorin, J Diener, BK Meyer, J Majewski, L Eckey , A Hoffman, H Amano, I Akasaki, K Hiramatsu, T Detchprohm, Phys. Rev. B 53, 16543-16550 (1996).

[16]Numerical Data and Functional Relationship in Science and Technology, edited by K.H. Hellwege, Landolt-Börnstein, New Series, Group III, Vol. 17, Subvol. a (Springer-Verlag, Berlin, 1982)

[17] W. Shan, T. J. Schmidt, R. J. Hauenstein, J. J. Song , B. Goldenberg , Appl. Phys. Lett. 66, 3492-3494 (1995).

[18] S. Chichibu , T. Azuhata, T. Sota , S. Nakamura, J. Appl. Phys. 79, 2784-2786 (1996).

[19] J. Petalas, S. Logothetidis, S. Boultadakis, M. Alouani, J. M. Wills , Phys. Rev. B 52, 8082-8091 (1995).

[20] W. Shan, T. J. Schmidt, X. H. Yang, S. J. Hwang, J. J. Song , B. Goldenberg , Appl. Phys. Lett. 66, 985-987 (1995).

[21] Zhongqin Yang, Zhizhong Xu, Phys. Rev. B 54, 17577 (1996).

[22] Masakatsu Suzuki, Takeshi Uenoyama, Akira Yanase, Phys. Rev. B 52, 8132-8139 (1995). 
[23] N Grandjean, J Massies, M Leroux, Appl. Phys. Lett. 69, 2071-2073 (1996).

[24] R. Cingolani, M. Ferrara, M. Lugara, G. Scamarcio, Sol. St. Comm. 58, 823 (1986).

[25] M. Smith, G. D. Chen, J. Y. Lin, H. X. Jiang , M. Asif Khan, C. J. Sun, Q. Chen, J. W. Yang , J. Appl. Phys. 79, $7001-7004$ (1996).

[26] G. D. Chen, M. Smith, J. Y. Lin, H. X. Jiang , Su-Huai Wei , M. Asif Khan, C. J. Sun , Appl. Phys. Lett. 68, 2784-2786 (1996).

[27] D. C. Reynolds, D. C. Look, W. Kim, O. Aktas, A. Botchkarev, A. Salvador, H. Morkoc, D. N. Talwar , J. Appl. Phys. 80, 594-596 (1996).

[28] Y. F. Tsay, B. Gong, S. S. Mitra, J. F. Vetelino, Phys. Rev. B 6, 2330 (1972).

[29] V. V. Sobolev, V. I. Donetskikh, E. F. Zagainov, Sov. Phys. Semicond. 12, 646 (1978).

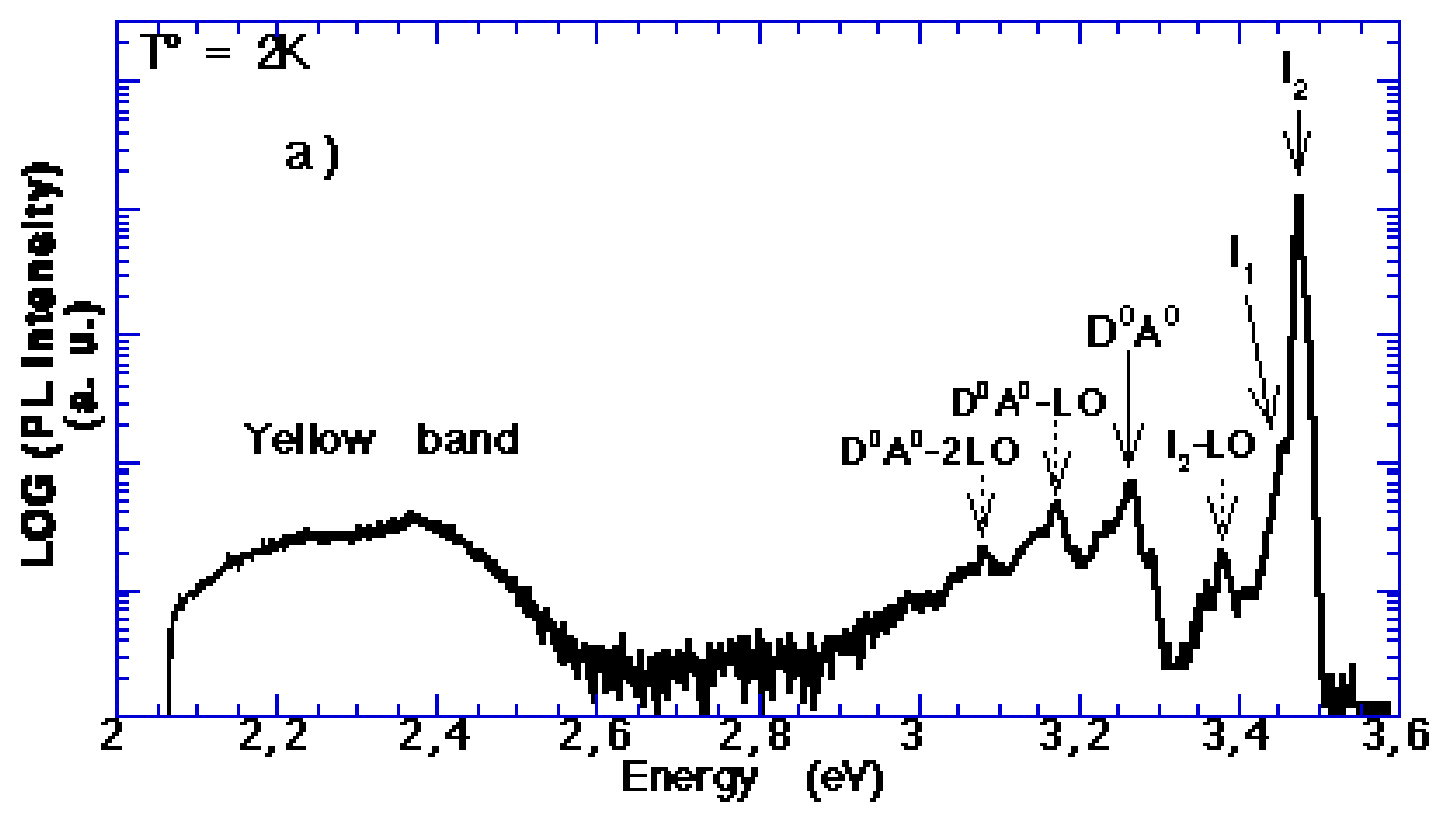

Figure 1a. Photoluminescence spectrum at low temperature $(T=2 K)$, the excitation energy is $E_{e x c}=4 \mathrm{eV}$.

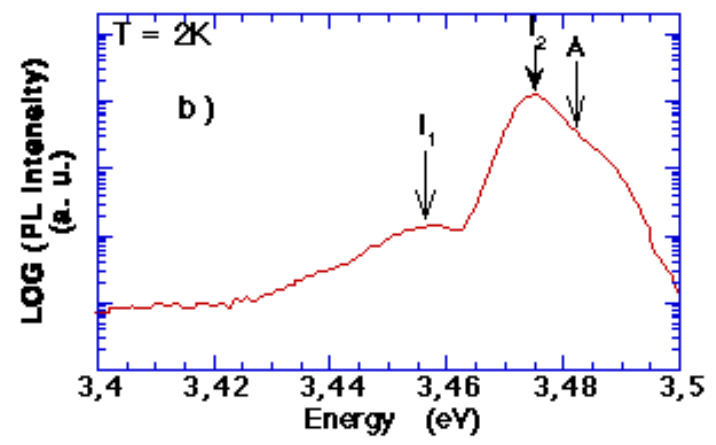

Figure 1b. Close-up on this photoluminescence spectrum in the range $3.4-3.5 \mathrm{eV}$. 


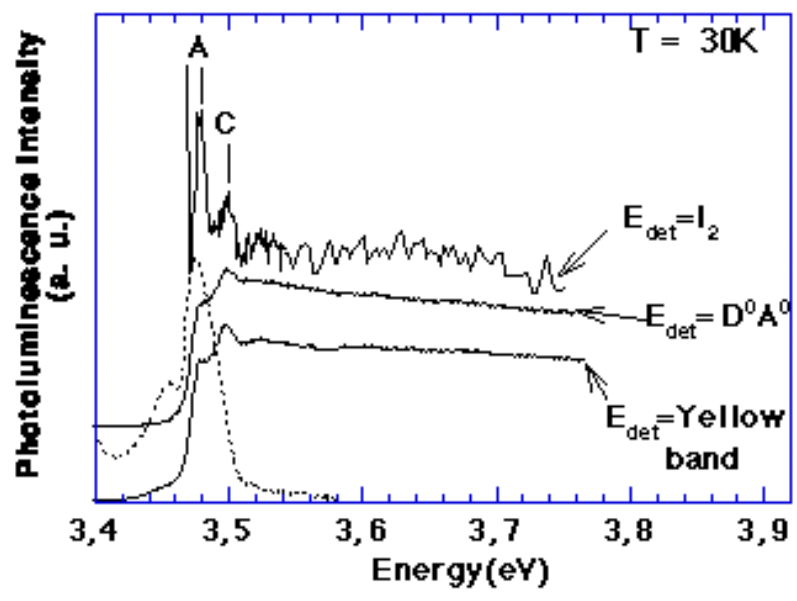

Figure 2. PL spectrum (dashed line) and PLE spectra (solid lines) detected on $\mathrm{I}_{2}: \mathrm{E}_{\text {det }}=\mathrm{I}_{2}=$ $3.457 \mathrm{eV}$, on $\mathrm{D}^{0}-\mathrm{A}^{0}: \mathrm{E}_{\text {det }}=\mathrm{D}^{0}-\mathrm{A}^{0}=3.26 \mathrm{eV}$, on the yellow band: $E_{\text {det }}=$ yellow band $=2.3 \mathrm{eV}$. The temperature is $T=30 \mathrm{~K}$. Spectra have been scaled and shifted for the sake of clarity.

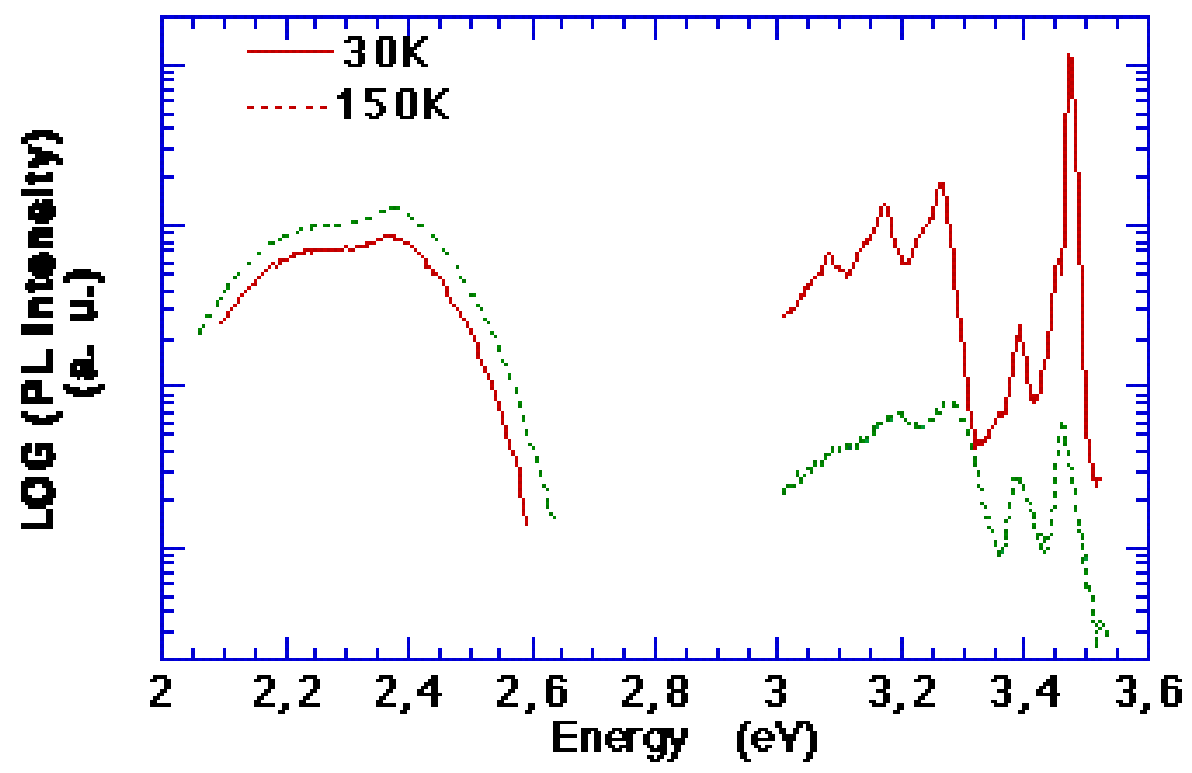

Figure 3. Photoluminescence spectrum at 30 (solid line) and 150K (dashed line). The excitation energy is 4 $\mathrm{eV}$. 


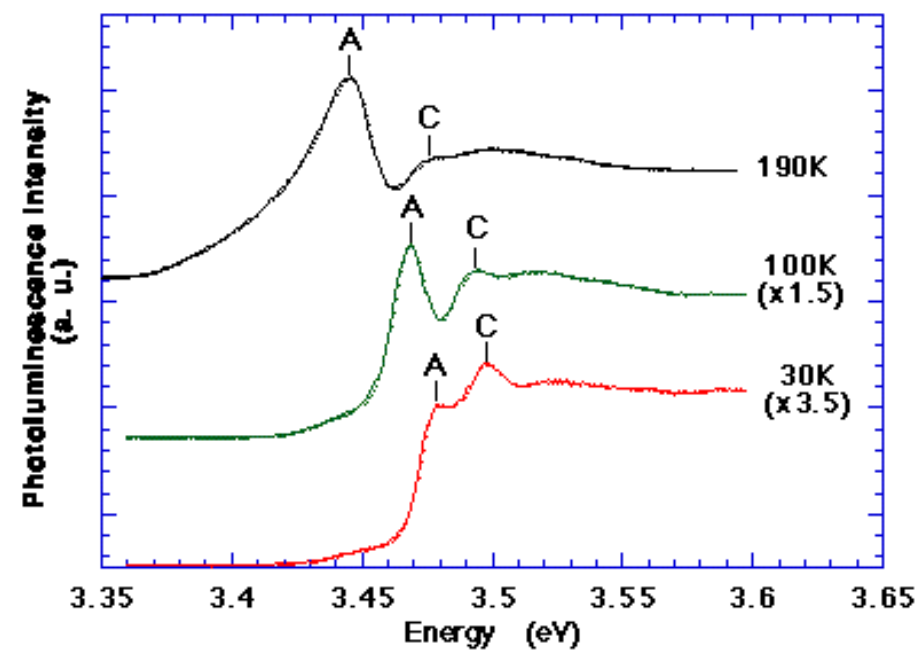

Figure 4. Photoluminescence excitation spectra detected at the energy of the yellow band $(2.37 \mathrm{eV})$ for 30,100 and $190 \mathrm{~K}$. Spectra have been scaled and shifted for the sake of clarity.

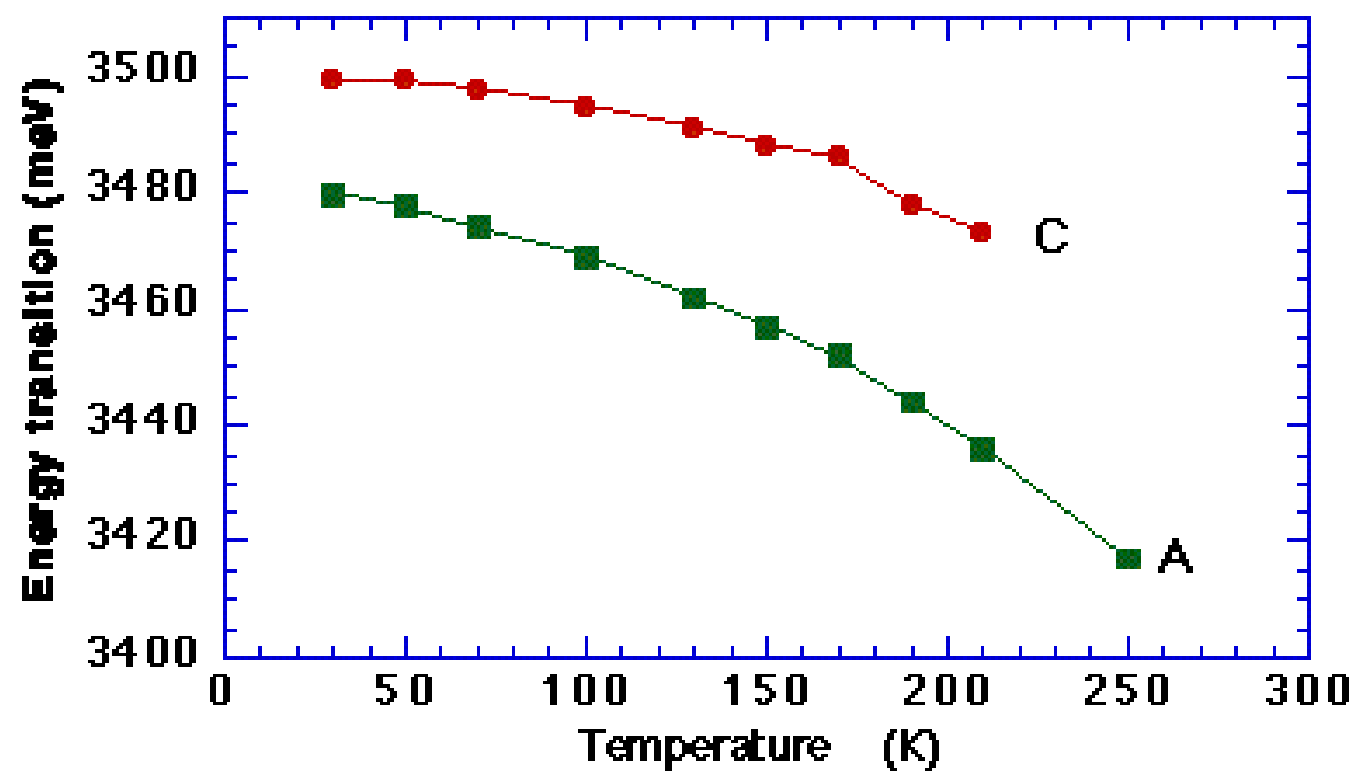

Figure 5. Energy positions of the observed peaks in our PLE spectra detected at the $D^{0} A^{0}$ line and at the yellow band, versus temperature.

(C) 1997 The Materials Research Society

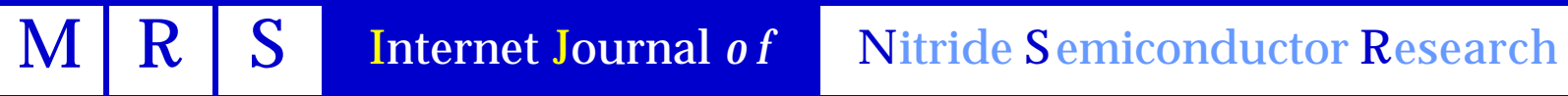

